\title{
A statistical study of galactic bright Be stars
}

\section{Anatoly S. Miroshnichenko}

Dept. of Physics \& Astronomy, University of North Carolina at Greensboro, P.O. Box 26170, Greensboro, NC 27402-6170, USA; email: a_mirosh@uncg.edu

\begin{abstract}
Creation of a modern database with a wide variety of data is an important step toward a better understanding of the Be phenomenon. In an effort to do that, I refined the existing catalog of Galactic Be stars and collected available observational data for 340 brightest objects and present some results of these data analysis. New candidates for Be binaries, which seem to represent a large fraction of Be stars, are suggested. Importance of the circumstellar optical continuum for modeling of the Be star envelopes is illustrated.
\end{abstract}

Keywords. stars: emission-line, Be, circumstellar matter

\section{Introduction}

The Be phenomenon is observationally defined as the presence of emission lines in the spectra of rapidly-rotating B-type stars. Stars with the Be phenomenon show continuum excess radiation (due to free-free and bound-free transitions in the circumstellar gas), variations in brightness and spectral lines, and periods of complete loss of the emission spectrum. They have disk-like envelopes with no dust and are though to be at the mainsequence evolutionary stage.

Among many catalogs of emission-line stars, only one intended to list Be stars alone (Jaschek \& Egret 1982). It contains very limited information, but can be used for creating a modern database. I collected IR fluxes (IRAS, 2MASS) for all catalog stars and other available data for 340 brightest ones, including 19 found since 1982 ( $V \leqslant 7.5 \mathrm{mag}$ ): binary status (detection, orbital period), polarization, parallax, rotational velocity, fundamental parameters, variability data (multicolor optical and near-IR photometry, $\mathrm{H} \alpha$ line).

The main results of my study of this material are as follows. Objects accessible from the northern hemisphere $\left(\delta \geqslant-20^{\circ}\right)$ have been better observed than more southern objects. Observations of $V \geqslant 8$ mag stars are needed for further constraining the list of Galactic
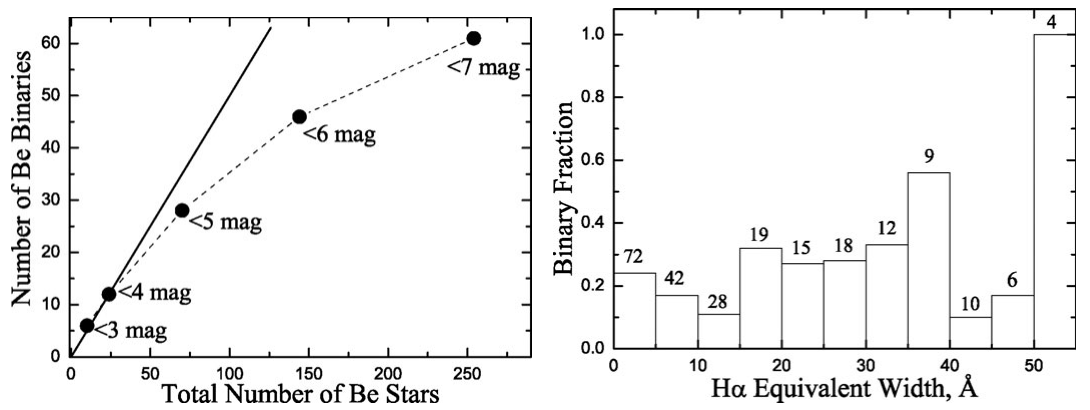

Figure 1. Left panel: Cumulative binary statistics for $254 \mathrm{Be}$ stars with $\mathrm{V}<7$ mag. Circles show the numbers of known binaries brighter than the indicated level. The solid line marks a $50 \%$ binary fraction. The number of recognized binaries used here is 61 . Right panel: Binary fractions are shown as a function of the $\mathrm{H} \alpha$ equivalent width (EW). The maximum $\mathrm{H} \alpha$ strength was used whenever possible. The total number of Be stars used in each bin is shown above it. 
Table 1. Statistics of the original catalog

\begin{tabular}{|l|r|}
\hline Category & Number \\
\hline Total number of objects (duplicates excluded) & 1149 \\
Herbig Ae/Be and Vega-type & 12 \\
Objects with the B [e] phenomenon and LBV & 10 \\
Supergiants and WR stars & 48 \\
Bright giants (luminosity type II) & 25 \\
Luminosity type II/III & 24 \\
Oe type stars & 4 \\
No line emission confirmed & 3 \\
Other unrelated objects & 2 \\
Objects with no luminosity type & 103 \\
Objects with no good line profiles & $>500$ \\
\hline
\end{tabular}
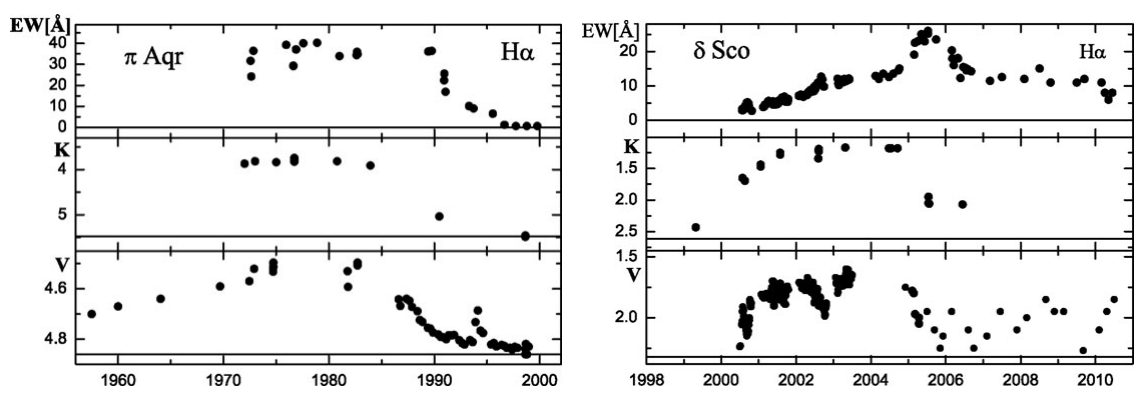

Figure 2. Brightness and $\mathrm{H} \alpha$ variations of $\pi$ Aqr (left) and $\delta$ Sco (right). Solid lines show the no-disk level of the parameters. Data for $\pi$ Aqr are taken from Bjorkman et al. (2002), for $\delta$ Sco from Carciofi et al. (2006), Halonen et al. (2008), Pollmann (2010), and Otero (see http://varsao.com.ar/delta_Sco.htm).

Be stars. The progressively lower observed binary fraction for fainter objects (Fig. 1, left) is a selection effect: fainter stars are less studied. Therefore, over $50 \%$ of Be stars can be binary systems. The right panel of Fig. 1 may be misleading, as not many stars have been observed extensively. However, many bright Be stars with strong $\mathrm{H} \alpha$ emission (e.g., $\gamma$ Cas, $\pi$ Aqr, $\chi$ Oph, $\varnothing$ Cas, 66 Oph) were recognized as binaries. Good candidates for the secondary companion search include: $\psi$ Per, HD 7636, HD 50083, EW Lac, V777 Cas, V659 Mon, NV Pup, MWC 19, HD 206773.

Fig. 2 shows brightening of two Be binaries during a strong line-emission phase. These data show that both mass loss and the disk structure are inhomogeneous. Modeling of emission-line profiles in stars with no edge-on disk that does not account for the optical excess radiation gives wrong results for the disk parameters. This effect also leads to an overestimated star's luminosity by up to 70\%. $\pi$ Aqr has a circular orbit with a 84.3-day period. Currently it shows weak signs of emission. The simultaneous optical fading and $\mathrm{H} \alpha \mathrm{EW}$ increase of $\delta$ Sco (orbital period 10.8 years - see Ames et al. in these proceedings, eccentricity $e=0.94$ ) in 2005 are due to dissipation of the disk inner part.

\section{References}

Jaschek, M. \& Egret, D. 1982, in: M. Jaschek \& H.-G. Groth (eds.), Be Stars, IAU Symposium 98, p. 261

Bjorkman, K. S., Miroshnichenko, A. S., McDavid, D., \& Pogrosheva, T. M. 2002, ApJ, 573, 812

Carciofi, A. C., Miroshnichenko, A. S., Kusakin, A. V., Bjorkman, J. E. et al. 2006, ApJ, 652, 1617

Halonen, R. J., Jones, C. E., Sigut, T. A. A., Zavala, R. T. et al. 2008, PASP, 120, 498

Pollmann, E. 2010, Be Star Newsletter, No. 40, in press 